\title{
PRODUÇÃo CIENTÍFICA BRASILEIRA EM FINANĢAS NO PERíodo 2000-2010
}

\author{
BRAZILIAN SCIENTIFIC PRODUCTION IN FINANCE IN THE PERIOD 2000-2010 \\ PRODUCCIÓN CIENTÍFICA BRASILEÑA SOBRE FINANZAS EN EL PERÍODO 2000-2010
}

\section{RESUMO}

Este artigo faz uma avaliação bibliográfica quantitativa e qualitativa dos artigos de Finanças publicados em 11 periódicos científicos nacionais, por meio de levantamentos sobre coautorias, áreas temáticas e uma análise da produtividade dos autores. A coautoria tornou-se uma característica predominante. As áreas temáticas mais comuns, finanças corporativas e gestão de investimentos, refletem a abundância de dados de empresas com ações em bolsa, negligenciando as demais. A produtividade é concentrada em poucos indivíduos no Sudeste e mais baixa do que o previsto pela teoria bibliométrica. A grande maioria dos autores publicou apenas um artigo e apenas 5\% publicaram cinco ou mais. A maior parte dos artigos internacionais dos autores prolíficos consta de periódicos de baixo impacto. A pouca inovação teórica e metodológica dificulta publicações internacionais de impacto. A avaliação da Coordenação de Aperfeiçoamento de Pessoal de Nível Superior (Capes) parece estimular parcerias, mas sugeriram-se novos incentivos visando ao alinhamento com o nível de desenvolvimento da área nos países mais avançados.

PALAVRAS-ChaVe Artigos científicos no Brasil, literatura de Finanças, produtividade dos autores em Finanças, periódicos de Finanças, Capes.

Ricardo Pereira Câmara Leal rleal@ufrj.br

Professor do Instituto Coppead de Administração, Universidade Federal do Rio de Janeiro - Rio de Janeiro - RJ, Brasil

Vinicio de Souza e Almeida almeida.vinicio@gmail.com

Professor do Departamento de Ciências Administrativas, Universidade Federal do Rio Grande do Norte - Natal - RN, Brasil

Patrícia Maria Bortolon p.m.bortolon@gmail.com

Professora do Departamento de Ciências Contábeis, Universidade Federal do Espírito Santo - Vitória - ES, Brasil

\begin{abstract}
This article conducts a quantitative and qualitative appraisal of the bibliography of financial articles published in 11 Brazilian scientific journals, through surveys into the question of co-authorships and other priority areas, together with an analysis of the productivity of authors. The issue of co-authorships became a dominant feature of the inquiry. The most common areas were corporate finance and investment management, and this reflects the large amount of data supplied by publicly traded companies, although it means that others were neglected. The production of literature is concentrated in the hands of a few people in the South-East of the country and is lower than what was predicted by the bibliometric theory. A large majority of authors only published one article and only $5 \%$ published five or more. The international articles of the most prolific authors are largely found in "low impact" periodicals. The lack of innovation, either of a theoretical or methodological kind, prevents international publications from having a significant effect. The assessments of seems to stimulate the formation of partnerships, but new incentives are recommended to ensure the level of development is aligned with that of more advanced countries. keywords Brazilian scientific articles, financial literature, authors on finance productivity, financial periodicals, Capes.
\end{abstract}

Resumen Este artículo hace una evaluación bibliográfica cuantitativa y cualitativa de los artículos sobre finanzas publicados en 11 periódicos cientificos nacionales, por medio de relevamientos sobre coautorías, áreas temáticas y un análisis de la productividad de los autores. La coautoría se tornó una característica predominante. Las áreas temáticas más comunes, finanzas corporativas y gestión de inversiones, refleja la abundancia de datos de empresas con acciones en bolsa, relegando las demás. La productividad es concentrada en pocos individuos en el Sudeste y más baja que lo previsto por la teoría bibliométrica. La gran mayoría de los autores publicó solo un artículo y apenas 5\% publicó cinco o más. La mayor parte de los artículos internacionales de Ios autores prolíicos consta en periódicos de bajo impacto. La poca innovación teórica y metodológica dificulta publicaciones internacionales de impacto. La evaluación Capes parece estimular alianzas, pero se sugirieron nuevos incentivos con vistas al alineamiento con el nivel de desarrollo del área en los paises más avanzados.

Palabras clave Artículos científicos en Brasil, literatura de finanzas, productividad de los autores de finanzas, periódicos de finanzas, Capes. 


\section{INTRODUÇÃo}

O crescimento econômico depende da escolha e do financiamento de bons projetos de investimento. $\mathrm{O}$ estudo de Finanças é relevante para se entender como os empreendimentos são avaliados e financiados, seus recursos financeiros e riscos são geridos e os mercados e instituições financeiras atuam ao intermediar as transações que os viabilizam. Brealey, Myers e Allen (2006, p. 5) afirmam que Finanças também considera o comportamento das pessoas, além de dinheiro e mercados.

A área de Finanças conta com estudiosos da Administração, Economia, Contabilidade, Engenharia de Produção, Matemática e Estatística, entre outras. O sistema de classificação do Journal of Economic Literature (JEL) enumera especialidades como Finanças de Empresas, Mercados Financeiros, Instituições e Serviços Financeiros e Governança Corporativa. Entender as características e a produtividade dos autores de artigos científicos de uma área de estudo contribui para seu desenvolvimento teórico e prático. Bertero, Caldas e Wood Jr. (2005) apresentaram um panorama da Administração com ênfase nos anos 1990. Os artigos brasileiros eram predominantemente derivativos, reproduzindo modelos estrangeiros, sua qualidade ainda era baixa, a despeito do aumento na quantidade, e havia poucos artigos de pesquisadores brasileiros em periódicos internacionais de alto impacto.

Leal, Oliveira e Soluri (2003) apresentaram um primeiro panorama da produção científica em Finanças no Brasil. Eles analisaram 551 artigos publicados entre 1974 e 2001 na Revista de Administração Contemporânea (RAC), RAE-Revista de Administração de Empresas (RAE), Revista de Administração da Universidade de São Paulo (RAUSP), Revista Brasileira de Economia $(R B E)$ e na extinta Revista Brasileira de Mercado de Capitais (RBMEC), além de 264 artigos incluídos nos Anais do Encontro Nacional da Associação Nacional de Pós-Graduação e Pesquisa em Administração (EnANPAD) no mesmo período. Ignorando os resultados obtidos para a $R B M E C$, eles concluíram que: (1) a maioria dos artigos apresentava somente um autor; (2) a produtividade era concentrada em poucos indivíduos e mais baixa do que sugere a teoria bibliométrica; (3) a maioria dos autores publicou apenas um artigo; (4) a maioria dos artigos era de instituições do Rio de Janeiro e São Paulo e todos os autores mais prolíficos eram das regiões Sul e Sudeste.
Ainda no campo de Finanças no Brasil, Camargos, Coutinho e Amaral (2005) analisaram 171 artigos publicados nos anais do EnANPAD entre 2000 e 2004. Eles verificaram que a maioria dos artigos tinha dois autores, em contraste com Leal, Oliveira e Soluri (2003), e que a maioria dos autores era do sexo masculino e do Rio de Janeiro e São Paulo. A maior parte dos artigos foi redigida em português, era empírica com emprego de dados secundários, apresentava bibliografia estrangeira dominante e se circunscrevia aos campos de finanças corporativas, derivativos, gestão de riscos e mercado de capitais. Matsumoto e outros (2008) constataram que boa parte dos artigos publicados em Finanças apresenta coautorias e sugerem que isso se deve à crescente complexidade dos trabalhos da área. Souza, Murcia e Borba (2010) indicam que os programas de pósgraduação brasileiros mais atuantes em Finanças são os das principais universidades do Rio de Janeiro e São Paulo, enquanto Iquiapaza, Amaral e Bressan (2009) argumentam que os artigos científicos em Finanças se tornaram mais normativos, quantitativos e próximos às ciências naturais e que a emersão do campo das finanças comportamentais pode ser uma alternativa a essa tendência.

O objetivo deste estudo é traçar um perfil dos artigos de Finanças publicados em periódicos brasileiros selecionados no período entre 2000 e 2010. $\mathrm{O}$ artigo apresenta uma avaliação das características dos artigos científicos sobre Finanças publicados no período e da produtividade de seus autores, considerando, particularmente, o surgimento de novos periódicos científicos com foco em Finanças e a ampliação do escopo de análise ao se incluírem mais revistas de Administração e de áreas correlatas, como Economia, Contabilidade e Engenharia de Produção. Finalmente, o artigo oferece uma análise qualitativa sobre a evolução da área e algumas sugestões para fortalecê-la.

A seção seguinte apresenta características da amostra de artigos de Finanças empregada, dando detalhes sobre os periódicos pesquisados e os critérios para composição da amostra de artigos de Finanças analisados. A seção principal do trabalho oferece informações quantitativas sobre artigos e autorias, trata da produção segundo subáreas temáticas e quantifica e discute a produtividade dos autores em Finanças. A conclusão oferece um sumário dos resultados e sugestões para fortalecer a produção científica nacional em Finanças. 


\section{METODOLOGIA}

\section{Seleção da amostra}

Os periódicos científicos nacionais pesquisados incluem aqueles ainda existentes, examinados por Leal, Oliveira e Soluri (2003), mais alguns periódicos adicionais da área de Administração e de áreas correlatas que publicam artigos de Finanças ocasionalmente. Os periódicos de áreas correlatas incluem somente aqueles classificados no estrato A2 pelos representantes da área de Administração na Capes em 2010. Os periódicos selecionados foram:

- Brazilian Administration Review (BAR), criada em 2004;

- Economia Aplicada (EA);

- Gestão \& Produção (GP);

- Revista Brasileira de Economia (RBE);

- Revista Brasileira de Finanças (RBFin), criada em 2003;

- Revista Contabilidade E Finanças ( $R C F$ ), que intitulava-se Caderno de Estudos até 2000;

- Revista de Administração Contemporânea (RAC), incluindo a RAC-eletrônica, publicada entre 2007 e 2009, quando foi fundida com a $R A C$;

- Revista de Administração Mackenzie (RAM);

- Revista de Administração da Universidade de São Paulo (RAUSP);

- RAE-Revista de Administração de Empresas (RAE), incluindo a RAE-eletrônica, publicada entre 2002 e 2010, quando foi fundida com a $R A E$, e

- $\quad$ Revista de Produção (PROD).

A RBFin foi criada em 2003 como o primeiro periódico científico brasileiro dedicado exclusivamente a Finanças. A extinta RBMEC não era unicamente uma revista científica, pois também atuava como publicação oficial do antigo Instituto Brasileiro de Mercado de Capitais (IBMEC) e publicava vários artigos de pesquisa e de opinião de seus quadros. A $R C F$ tem como foco principal a área de Contabilidade. Souza, Murcia e Borba (2010) apresentam os resultados de uma pesquisa com 20 docentes de Finanças vinculados a programas de doutorado sobre os periódicos brasileiros que consideravam mais importantes. A RBFin aparece em primeiro lugar, segundo 14 deles, seguida da RAE, RAUSP, RBE, RCF e $R A C$. Uma nova revista dedicada à área de Finanças, a Revista de Finanças Aplicadas, criada em 2010, não foi incluída na análise por não ter histórico suficiente.

\section{Modelo bibliométrico}

O grau de desenvolvimento de uma área do conhecimento se reflete na produtividade de seus autores. A quantidade de artigos de Finanças por autor e por periódico foi analisada por meio da "Lei de Lotka" (CHUNG e COX, 1990), que responde à seguinte pergunta: "Dado que $x$ autores publicaram um artigo, quantos autores publicariam $n$ artigos?". A previsão oferecida pelo modelo é retratada pela Equação 1, onde $a_{n}$ é o número de autores que publicaram $n$ artigos, $a_{1}$ é o número de autores que publicaram um artigo e $c$ é a constante que Lotka estimou ser aproximadamente dois. Logo, se 100 autores publicaram um artigo em certa área do conhecimento, então quatro autores (100/52) publicariam cinco artigos.

$$
a_{n}=\frac{a_{1}}{n^{c}}
$$

A constante $c$ pode, então, ser estimada para cada periódico de Finanças e para todos eles conjuntamente, uma vez que $a_{1}$ e diversos $a_{n}$ foram obtidos dos periódicos nacionais selecionados. A Equação 2 retrata o modelo empírico estimado forçando intercepto nulo, no qual $\varepsilon$ é o erro da regressão. A Equação 2 é o logaritmo da Equação 1, de modo que $c$ possa ser o coeficiente estimado via regressão linear simples. A Equação 1 permite ver que um coeficiente $c$ maior do que dois implica que a quantidade de autores produzindo mais do que um artigo seria menor do que o esperado quando $c=2$, sugerindo uma baixa produtividade. Chung e Cox (1990) demonstraram, para artigos de Finanças e para $c=2$, que o número de autores com apenas um trabalho publicado deveria ser igual a $\sigma / \pi^{2}$ por cento $(\pi=3,1416 \ldots)$ do total de autores, ou $60,79 \%$. A proporção do total de autores seria de $\sigma /(n \pi)^{2}$ para qualquer quantidade $n$ de artigos publicados em Finanças.

$$
\log \left(\frac{a_{n}}{a_{1}}\right)=-c \times \log (n)+\varepsilon
$$

\section{RESULTADOS}

\section{Análise descritiva da amostra}

A Tabela 1 mostra que 3.417 artigos foram objeto da análise inicial, sendo 461 (14\%) classificados como 
de Finanças, considerando o conteúdo de seu título, resumo e palavras-chave e seu enquadramento em uma das subáreas temáticas empregadas, definidas mais adiante. Todos os artigos publicados na RBFin foram automaticamente considerados como de Finanças. Os artigos cuja classificação seria mais questionável, por serem fronteiriços com as áreas de Contabilidade e de Economia, não foram incluídos. A maioria dos artigos veio, naturalmente, da RBFin, porque ela publica exclusivamente artigos de Finanças. Contudo, é interessante notar que a RAUSP supera a $R C F$ no número de artigos de Finanças. Os periódicos da área de Engenharia de Produção, GP e $P R O D$, foram os que menos contribuíram para a amostra, enquanto os de Administração publicaram mais artigos de Finanças do que os de Economia, com exceção da $B A R$.

As únicas revistas que publicaram artigos de Finanças em todos os anos do período de análise foram a RAUSP, a $R C F$ e a $R A C$, além da RBFin, obviamente. Aparentemente, houve uma queda no número de artigos de Finanças publicados na $R A C$, RAUSP e RAE, o que pode ser reflexo do aumento no número de artigos publicados na RBFin. É possível que uma gradual especialização dos periódicos esteja ocorrendo. Contudo, essa conclusão é precá- ria, porque o período amostral é curto. O número de artigos publicados em inglês aumentou um pouco, ficando entre $15 \%$ e $16 \%$ do total dos artigos de Finanças nos últimos anos do período estudado. O quantitativo anual de artigos por periódico pode ser obtido com os autores.

O número mais comum de autores é dois. É mais comum haver três autores do que um autor, e a ocorrência de quatro autores também não é desprezível nos periódicos de Administração, conforme a Tabela 2. Não há artigos com mais do que três autores nos periódicos de Economia ( $R B E$ e $E A$ ), e a ocorrência de um autor ainda é mais comum do que a de três autores na RBE. Leal, Oliveira e Soluri (2003) constataram que a maioria dos artigos tinha um autor entre 1974 e 2001. O aumento da coautoria pode ser mais acentuado entre os autores de Administração e estar relacionado ao sistema de atribuição de pontos aos periódicos pela Capes. Caso muitos artigos ainda resultem de trabalhos de conclusão de curso, a inclusão do orientador do trabalho e demais membros da banca entre os autores pode ter se tornado comum, visando à maior acumulação de pontos. É possível, também, que a competição por avaliações melhores e por mais recursos públicos para a pesquisa possa ter estimulado parcerias para aprimorar a qualidade

\section{Tabela 1 - Número de exemplares, total de artigos e de artigos de Finanças entre 2000 e 2010}

\begin{tabular}{|l|c|c|c|c|c|}
\hline \multicolumn{1}{|c|}{ Periódico } & $\begin{array}{c}\text { Número } \\
\text { de artigos }\end{array}$ & $\begin{array}{c}\text { Número } \\
\text { de exemplares }\end{array}$ & $\begin{array}{c}\text { Artigos } \\
\text { por exemplar }\end{array}$ & $\begin{array}{c}\text { Número de artigos } \\
\text { de Finanças }\end{array}$ & $\begin{array}{c}\% \text { dos } 461 \text { artigos } \\
\text { de Finanças }\end{array}$ \\
\hline BAR & 106 & 20 & 5 & 11 & 2 \\
\hline EA & 324 & 44 & 7 & 18 & 4 \\
\hline GP & 407 & 35 & 12 & 9 & 2 \\
\hline PROD & 336 & 31 & 11 & 6 & 1 \\
\hline RAC & 523 & 61 & 9 & 56 & 12 \\
\hline RAE & 486 & 62 & 8 & 49 & 6 \\
\hline RAM & 274 & 40 & 7 & 26 & 21 \\
\hline RAUSP & 337 & 44 & 8 & 98 & 23 \\
\hline RBE & 271 & 44 & 6 & 22 & 13 \\
\hline RBFin & 105 & 21 & 5 & 105 & 100 \\
\hline RCF & 248 & 34 & 7 & 61 & 23 \\
\hline Todos & 3.417 & 436 & 8 & 461 & \\
\hline
\end{tabular}

Nota: 0 significado dos acrônimos dos nomes dos periódicos encontra-se no texto. 
dos artigos, como apontado por Matsumoto e outros (2008).

\section{Subáreas temáticas}

As subáreas temáticas de Finanças do Enanpad influenciaram nossas categorias de classificação dos artigos, que permitiu identificar os grandes tópicos mais frequentes e compará-los com a evidência nacional e internacional anterior. A Tabela 3 mostra as subáreas com maior número de artigos no período. O tema "finanças corporativas" corresponderia a $42 \%$ do total de artigos, se a ele agregássemos "governança corporativa" (GC) e "fusões e aquisições" (F\&A). Bancos de dados existentes no Brasil com informações de mercado, como o Economática ${ }^{\circledR}$ e Bloomberg ${ }^{\circledR}$, podem favorecer o destaque para o tema "apreçamento de ativos e gestão de carteiras", enquanto o tema F\&A talvez apresente a menor quantidade de artigos porque muitas operações não envolvem empresas com ações em bolsa, dificultando o acesso a dados. A preferência por artigos empíricos se reflete na maior incidência de artigos em subáreas favorecidas pela disponibilidade de dados. Esse padrão é similar ao constatado por Borokhovich, Bricker e Simkins (1994) para os EUA e Camargos, Coutinho e Amaral (2005) para os trabalhos de Finanças apresentados no Enanpad. O tema GC recebeu mais atenção há alguns anos. É interessante notar que também se publica pouco sobre mercados e instituições finan- ceiras e econometria financeira, as subáreas que poderiam ser mais desenvolvidas no País.

Analisamos os artigos publicados na RBFin como amostra representativa dos artigos nacionais de Finanças. Sua principal fraqueza pode ser a baixa inovação em termos teóricos e metodológicos, uma vez que a maioria reproduz testes empíricos internacionais com dados nacionais. Os artigos abordam empresas com ações em bolsa, cujas informações são abundantes e pouco oferecem sobre as demais empresas. A área apresenta certo descolamento da realidade local nesse aspecto. Esses resultados sustentam as conclusões de Bertero, Caldas e Wood Jr. (2005).

\section{Produtividade dos autores}

O desenvolvimento de uma área se reflete na produtividade de seus autores. O emprego de um modelo bibliométrico de produtividade permite a análise evolutiva relativa a estudos anteriores. A amostra revelou 643 autores diferentes. A Tabela 4 apresenta sua frequência de publicação segundo a quantidade de artigos agregada e em cada periódico. Somente $22 \%$, entre todos os autores, publicaram mais do que um artigo, e apenas $7 \%$ publicaram quatro ou mais artigos no período. Os percentuais são praticamente iguais aos de Leal, Oliveira e Soluri (2003) no período 1974-2001. A produtividade é baixa se comparada à previsão do modelo bibliométrico e à dos autores dos

\section{Tabela 2 - Percentual de artigos segundo o número de autores}

\begin{tabular}{|c|c|c|c|c|c|c|c|}
\hline \multirow{2}{*}{ Periódicos } & \multicolumn{6}{|c|}{$\mathrm{N}^{0}$ de autores por artigo (\%) } & \multirow{2}{*}{$\mathrm{N}^{0}$ de artigos } \\
\hline & 1 & 2 & 3 & 4 & 5 & 6 & \\
\hline$B A R$ & 0 & 82 & 9 & 9 & 0 & 0 & 11 \\
\hline$E A$ & 28 & 44 & 28 & 0 & 0 & 0 & 18 \\
\hline$G P$ & 0 & 67 & 22 & 11 & 0 & 0 & 9 \\
\hline PROD & 17 & 67 & 17 & 0 & 0 & 0 & 6 \\
\hline$R A C$ & 16 & 52 & 25 & 5 & 2 & 0 & 56 \\
\hline$R A E$ & 27 & 47 & 18 & 8 & 0 & 0 & 49 \\
\hline RAM & 15 & 42 & 35 & 8 & 0 & 0 & 26 \\
\hline RAUSP & 19 & 46 & 29 & 6 & 0 & 0 & 98 \\
\hline RBE & 32 & 50 & 18 & 0 & 0 & 0 & 22 \\
\hline RBFin & 12 & 54 & 23 & 10 & 1 & 0 & 105 \\
\hline$R C F$ & 11 & 48 & 28 & 8 & 3 & 2 & 61 \\
\hline Todos & 17 & 50 & 25 & 7 & 1 & 0 & 461 \\
\hline $\mathrm{N}^{0}$ de artigos & 78 & 232 & 114 & 32 & 4 & 1 & - \\
\hline
\end{tabular}

Notas: (1) percentual de artigos de Finanças com $n$ autores relativo ao total de artigos de Finanças de cada periódico, em que $n$ varia de 1 a 6; (2) os percentuais foram arredondados e podem não somar 100\%; (3) o significado dos acrônimos dos nomes dos periódicos encontra-se no texto. 
EUA, conforme Chung e Cox (1990). A RAUSP foi a revista que apresentou menor percentual de autores com apenas um artigo de Finanças (79\%). Três dos quatro periódicos que não são de Administração ( $E A$, $G P$ e $P R O D)$ apresentaram os maiores percentuais de autores com apenas um artigo de Finanças.

A proporção de autores que publica apenas um artigo é maior e a produtividade na área de Finanças no Brasil é menor do que o previsto pela teoria bibliométrica, porque todas as constantes $c$ estimadas são superiores a dois. A constante $c$ estimada para todos os periódicos agregados $(2,63)$ ficou próxima àquela obtida por Leal, Oliveira e Soluri $(2003)(2,44)$, sugerindo que não houve melhora em relação ao período entre 1974 e 2001. É possível suspeitar que boa parte dos autores seja egressa de mestrados, que nunca mais publicaram, e que a piora na produtividade reflita o aumento no número de mestres e de mestrados. Testes de chi-quadrado também demonstraram que a distribuição de frequência dos periódicos agregados não acompanha a previsão para a área sugerida por Chung e Cox (1990).

\section{Autores prolíficos}

A identificação dos autores que mais publicam na área de Finanças é um caminho natural de investigação sobre a produtividade dos autores. Os autores prolíficos são os que publicaram cinco ou mais artigos no período da amostra. Houve 33 autores prolíficos, que correspondem a $5 \%$ do total de autores. Constata-se grande concentração da produção em poucos autores porque há pelo menos um autor prolífico em 57\% (263) dos artigos. A proporção de autores prolíficos não aumentou em relação ao período 1974-2001 no qual Leal, Oliveira e Soluri (2003) identificaram que $7 \%$ dos autores de Finanças publicaram mais do que cinco artigos. Vinte dos 33 autores prolíficos publicaram em quatro ou mais periódicos diferentes, entre os analisados. Os periódicos que mais publicaram artigos de autores prolíficos foram a RAUSP (75), a RBFin (46), a $R A C$ (38) e a $R A E(36)$. A Tabela 5 sugere uma correlação entre instituições e periódicos a elas vinculados. As publicações de autores prolíficos da Universidade de São Paulo (USP) ocorrem em maior número na RAUSP e $R C F$, e da Universidade Presbiteriana Mackenzie (UPM), na $R A M$ e na RAUSP. Por outro lado, os autores da FGV em São Paulo publicaram mais na RAUSP. As tabelas com a análise sobre os autores prolíficos estão disponíveis com os autores.

A Tabela 5 mostra que há enorme concentração da produção dos autores prolíficos nos estados de São

\section{Tabela 3 - Percentual de artigos por ano segundo suas áreas temáticas}

\begin{tabular}{|c|c|c|c|c|c|c|c|c|}
\hline Ano & AGC & FC & GC & GRD & MIF & EMN & F\&A & $\begin{array}{c}\mathrm{N}^{0} \mathrm{de} \\
\text { artigos no ano }\end{array}$ \\
\hline 2000 & 79 & 0 & 7 & 14 & 0 & 0 & 0 & 14 \\
\hline 2001 & 38 & 23 & 8 & 19 & 4 & 4 & 4 & 26 \\
\hline 2002 & 23 & 23 & 17 & 13 & 17 & 7 & 0 & 30 \\
\hline 2003 & 28 & 31 & 17 & 22 & 0 & 3 & 0 & 36 \\
\hline 2004 & 14 & 20 & 30 & 20 & 9 & 7 & 0 & 44 \\
\hline 2005 & 19 & 26 & 14 & 21 & 12 & 7 & 2 & 43 \\
\hline 2006 & 32 & 23 & 17 & 9 & 11 & 4 & 4 & 47 \\
\hline 2007 & 21 & 21 & 21 & 21 & 6 & 6 & 4 & 52 \\
\hline 2008 & 21 & 32 & 9 & 13 & 20 & 4 & 0 & 56 \\
\hline 2009 & 16 & 31 & 17 & 13 & 16 & 5 & 3 & 64 \\
\hline 2010 & 39 & 14 & 10 & 10 & 10 & 14 & 2 & 49 \\
\hline Todos & 26 & 24 & 16 & 16 & 11 & 6 & 2 & 461 \\
\hline $\mathrm{N}^{0}$ artigos & 119 & 111 & 73 & 72 & 49 & 27 & 10 & - \\
\hline
\end{tabular}

Nota: as áreas temáticas são "apreçamento de ativos e gestão de carteiras" (AGC), "Finanças corporativas" (FC), "governança corporativa" (GC), "gestão de riscos e derivativos" (GRD), "mercados e instituições financeiras" (MIF), "econometria e métodos numéricos em Finanças” (EMN) e "fusões e aquisições” (F\&A). 
Paulo e Rio de Janeiro. Metade das publicações é de autores vinculados a três instituições de São Paulo: USP, FGV-SP e UPM. Cerca de 16\% correspondem à Pontifícia Universidade Católica do Rio de Janeiro (PUC) e à Universidade Federal do Rio de Janeiro (UFRJ). A soma de todas as instituições das regiões Sul e Sudeste atinge $92 \%$ dos artigos de autores prolíficos. As instituições do Distrito Federal foram as únicas instituições com autores prolíficos fora dessas regiões. O Banco Central do Brasil (BC), a Universidade Nacional de Brasília (UNB) e a Universidade Católica de Brasília (UCB) contribuíram com $8 \%$ do total de artigos de autores prolíficos.

Os artigos internacionais dos autores prolíficos foram identificados em seus Currículos Lattes e somam 230. Praticamente todos os autores tiveram publicações internacionais no período. Cinco dos 33 autores prolíficos apresentam mais publicações internacionais do que nacionais. Apenas cinco dos 10 autores mais prolíficos nas publicações nacionais selecionadas mantém-se entre os 10 primeiros, se considerado o total de artigos nacionais e internacionais. A pesquisa sobre Finanças produzida no Brasil vem encontrando espaço nas publicações internacionais, mas a maioria dos autores prolíficos privilegia periódicos nacionais. Houve um total de sete artigos de autores prolíficos em periódicos internacionais classificados como A1 e 24 como A2 pela Capes. Setenta e quatro por cento dos artigos internacionais dos autores prolíficos foram publicados em periódicos do estrato B3 ou pior.

\section{CONCLUSÕES}

Este estudo analisou 461 artigos de Finanças, publicados em 11 periódicos científicos nacionais das áreas de Administração, Contabilidade, Economia e Produção, no período entre 2000 e 2010. Os artigos de Finanças representaram $14 \%$ do total de 3.417 artigos publicados nesses periódicos no período. A RBFin, a RAUSP e a $R C F$ publicaram, nessa ordem, a maior parte dos artigos de Finanças.

A maioria dos artigos foi classificada como de Finanças corporativas ou de gestão de investimentos e abordou empresas com ações em bolsa, devido à existência de bancos de dados confiáveis e de fácil acesso. O tema "fusões e aquisições", por outro lado,

\section{Tabela 4 - Percentual de autores segundo a quantidade de artigos publicados em cada periódico}

\begin{tabular}{|c|c|c|c|c|c|c|c|c|c|c|c|c|c|c|}
\hline \multirow{2}{*}{ Periódico } & \multicolumn{10}{|c|}{ Número de artigos publicados (\%) } & \multirow{2}{*}{$\begin{array}{l}\text { Total de } \\
\text { autores }\end{array}$} & \multirow{2}{*}{ c } & \multirow{2}{*}{$\mathbf{R}^{2}$} & \multirow{2}{*}{$\chi^{2}$} \\
\hline & 1 & 2 & 3 & 4 & 5 & 6 & 7 & 8 & 9 & $10+$ & & & & \\
\hline$B A R$ & 81 & 19 & 0 & 0 & 0 & 0 & 0 & 0 & 0 & 0 & 21 & $2,10^{\star}$ & 1,00 & 4,33 \\
\hline$E A$ & 97 & 0 & 3 & 0 & 0 & 0 & 0 & 0 & 0 & 0 & 34 & $3,17^{\star}$ & 1,00 & $15,37^{*}$ \\
\hline GP & 95 & 5 & 0 & 0 & 0 & 0 & 0 & 0 & 0 & 0 & 21 & $4,33^{*}$ & 1,00 & 8,33 \\
\hline PROD & 100 & 0 & 0 & 0 & 0 & 0 & 0 & 0 & 0 & 0 & 12 & NA & NA & 6,42 \\
\hline$R A C$ & 90 & 7 & 2 & 1 & 0 & 0 & 0 & 0 & 0 & 0 & 110 & $3,44^{*}$ & 0,99 & $29,04^{*}$ \\
\hline$R A E$ & 81 & 12 & 5 & 1 & 1 & 0 & 0 & 0 & 0 & 0 & 78 & $2,71^{*}$ & 0,99 & 7,48 \\
\hline$R A M$ & 90 & 4 & 4 & 2 & 0 & 0 & 0 & 0 & 0 & 0 & 52 & $3,04^{*}$ & 0,96 & $14,30^{*}$ \\
\hline RAUSP & 79 & 12 & 6 & 1 & 1 & 0 & 1 & 0 & 1 & 0 & 156 & $2,54^{*}$ & 0,99 & $14,18^{*}$ \\
\hline$R B E$ & 86 & 11 & 3 & 0 & 0 & 0 & 0 & 0 & 0 & 0 & 35 & $3,04^{*}$ & 0,99 & 6,87 \\
\hline RBFin & 83 & 8 & 6 & 3 & 0 & 0 & 0 & 0 & 0 & 0 & 192 & $2,58^{*}$ & 0,99 & $26,94^{*}$ \\
\hline$R C F$ & 83 & 12 & 3 & 1 & 0 & 1 & 0 & 0 & 0 & 0 & 121 & $3,13^{*}$ & 0,99 & $19,17^{*}$ \\
\hline Todos & 78,2 & 10,1 & 4,5 & 2,0 & 1,2 & 0,8 & 0,9 & 0,5 & 0,6 & 1,1 & 643 & $2,63^{*}$ & 0,99 & $56,98^{*}$ \\
\hline Previsto & 60,8 & 15,2 & 6,8 & 3,8 & 2,4 & 1,7 & 1,2 & 1,0 & 0,8 & 6,4 & - & - & - & - \\
\hline
\end{tabular}

Notas: (1) o número em cada célula é o percentual de autores com $n$ artigos de Finanças para o periódico em relação ao total de autores de Finanças que publicaram no periódico, por exemplo:

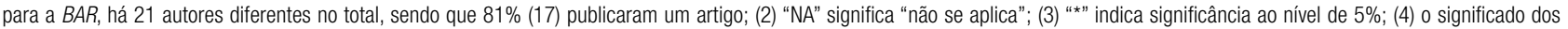
acrônimos dos nomes dos periódicos encontra-se no texto; (5) o valor crítico do teste de $\chi^{2}$ a $5 \%$ de significância é igual a 9,49; (6) a linha "Todos" apresenta a frequência de publicação considerando todas as publicações agregadas e a contagem nela apresentada; naturalmente, não corresponde ao somatório das linhas acima; (7) na linha "Todos" sob a coluna "10+", foram agregados dois autores com 11, um com 14, um com 15 e um com 18 artigos, para economia de espaço; (8) a última linha apresenta a distribuição de frequência prevista conforme a expressão $6 /(n \pi)^{2}$ definida por Chung e Cox (1990), e as probabilidades sob a coluna "10+" foram somadas para economia de espaço. 
envolve muitas empresas sem ações negociadas em bolsa e é pouco estudado. Há poucos artigos sobre métodos numéricos e econométricos em Finanças. A principal fraqueza dos artigos é a pouca inovação em termos teóricos e metodológicos, uma vez que grande parte deles replica trabalhos empíricos estrangeiros, dificultando sua publicação em periódicos internacionais influentes. A área apresenta certo descolamento da realidade nacional ao não privilegiar as empresas de capital fechado, cujos dados são difíceis de obter e menos confiáveis, e apresenta atraso em relação ao desenvolvimento científico nos países mais avançados.

A produtividade permaneceu concentrada em poucos indivíduos e nas instituições das regiões Sudeste e Sul, como em estudos anteriores, e é mais baixa do que o sugerido pela teoria bibliométrica e pela evidência empírica da área de Finanças nos EUA. A maioria dos autores publicou apenas um artigo. $\mathrm{O}$ percentual de autores que publicaram mais do que um artigo (22\%) ficou bem abaixo do previsto pela teoria bibliométrica (38\%). O número de autores prolíficos, definidos como os que publicaram cinco ou mais artigos, representa apenas 5\% do total de 643 autores e também é bem menor do que a teoria bibliométrica e a evidência nos EUA preveem. A grande proporção de autores que só publicam uma vez sugere que muitos artigos ainda derivam de dissertações de mestrado, um curso que cada vez mais tem ênfase na formação profissional e, portanto, podem não ser estudos científicos de ponta.

A produção científica de Finanças no Brasil ainda se encontra muito concentrada nos autores prolíficos, que estiveram entre os que escreveram 57\% dos artigos da amostra. Os autores prolíficos em periódicos nacionais publicaram mais no exterior, em comparação com estudos nacionais anteriores. Embora quase todos apresentassem publicações internacionais, apenas sete apresentaram mais publicações internacionais do que nacionais. Não houve uma publicação sequer nos quatro periódicos de maior impacto em Finanças classificados como A+ por Currie e Pandher (2011): The Journal of Finance, The Review of Financial Studies, Journal of Financial Economics e Journal of Financial and Quantitative

\section{Tabela 5 - Número de artigos de Finanças publicados em periódicos nacionais selecionados segundo o vínculo profissional dos autores que publicaram mais do que cinco artigos entre 2000 e 2010}

\begin{tabular}{|c|c|c|c|c|c|c|c|c|c|c|c|c|c|c|c|}
\hline \multirow[b]{2}{*}{ Periódicos } & \multicolumn{14}{|c|}{ Instituições (unidades da federação) } & \multirow[b]{2}{*}{ Total } \\
\hline & क् & $\frac{\text { एீ}}{\text { 己 }}$ & $\begin{array}{l}\frac{\tilde{\alpha}}{\tilde{b}} \\
\frac{\hat{g}}{b}\end{array}$ & $\frac{\overrightarrow{0}}{\mathfrak{2}}$ & $\begin{array}{l}\text { ญू } \\
\text { 플 }\end{array}$ & 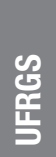 & 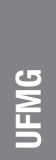 & 를 & 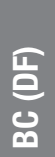 & 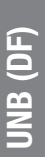 & 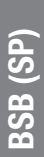 & $\begin{array}{c}\frac{\sigma}{3} \\
\frac{\text { 늘 }}{3}\end{array}$ & 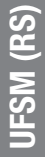 & 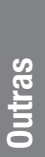 & \\
\hline$B A R$ & 3 & 2 & 1 & 0 & 0 & 0 & 0 & 0 & 1 & 2 & 0 & 0 & 0 & 0 & 9 \\
\hline$E A$ & 0 & 0 & 0 & 0 & 0 & 1 & 0 & 0 & 3 & 0 & 0 & 0 & 0 & 0 & 4 \\
\hline GP & 1 & 0 & 0 & 0 & 1 & 0 & 0 & 0 & 0 & 0 & 0 & 0 & 0 & 0 & 2 \\
\hline$R A C$ & 9 & 2 & 1 & 5 & 4 & 2 & 4 & 5 & 0 & 2 & 0 & 1 & 2 & 1 & 38 \\
\hline$R A E$ & 8 & 8 & 3 & 1 & 2 & 1 & 7 & 3 & 0 & 0 & 0 & 2 & 0 & 1 & 36 \\
\hline$R A M$ & 1 & 0 & 8 & 4 & 1 & 0 & 1 & 0 & 0 & 0 & 0 & 0 & 0 & 0 & 15 \\
\hline RAUSP & 30 & 11 & 8 & 3 & 2 & 2 & 5 & 6 & 1 & 0 & 3 & 0 & 2 & 2 & 75 \\
\hline$R B E$ & 0 & 0 & 0 & 0 & 2 & 4 & 0 & 2 & 0 & 0 & 1 & 0 & 0 & 0 & 9 \\
\hline RBFIN & 7 & 8 & 2 & 7 & 4 & 5 & 0 & 2 & 7 & 0 & 1 & 2 & 0 & 1 & 46 \\
\hline$R C F$ & 15 & 0 & 6 & 3 & 0 & 0 & 1 & 0 & 0 & 3 & 0 & 0 & 0 & 0 & 28 \\
\hline Todos & 74 & 31 & 29 & 23 & 16 & 15 & 18 & 18 & 12 & 7 & 5 & 5 & 4 & 5 & 262 \\
\hline \% Total & 30 & 12 & 12 & 9 & 6 & 6 & 6 & 5 & 5 & 3 & 2 & 2 & 2 & 2 & 100 \\
\hline
\end{tabular}

Nota: (1) 0 significado das siglas das instituições encontra-se no texto, exceto Universidade Federal de Santa Catarina (UFSC), Universidade Federal do Rio Grande do Sul (UFRGS), Universidade Federal de Minas Gerais (UFMG), Banco Sudameris Brasil (BSB), Universidade Federal de Juiz de Fora (UFJF) e Universidade Federal de Santa Maria (UFSM); (2) outras: UCB/DF (2 artigos na RAUSP); Faculdade Novos Horizontes/MG (1 artigo na RAC e 1 na RAE) e lbmec/SP (1 artigo na RBFin); (3) o nome IBMEC deriva-se da sigla de sua instituição antecessora, o Instituto Brasileiro de Mercado de Capitais; (4) o significado dos acrônimos dos nomes dos periódicos encontra-se no texto; (5) contou-se apenas a primeira instituição declarada por cada autor quando mais de um vínculo foi declarado; (6) não houve publicação de prolíficos na PROD. 
Analysis. Um exame das publicações internacionais dos autores prolíficos em periódicos nacionais revela que cerca de $13 \%$ delas encontram-se em periódicos do estrato A1 e A2 da Capes, enquanto $74 \%$ se deram em periódicos do estrato $\mathrm{B} 3$ ou pior.

Os resultados sugerem que houve alguma evolução qualitativa da pesquisa em Finanças no Brasil, mas que ela poderia ser melhor. A produtividade dos autores continua baixa, há poucos artigos em inglês (15\%) para facilitar a disseminação internacional, os autores prolíficos publicam relativamente pouco no exterior e, na maioria das vezes, em periódicos de pouca expressão.

Os autores brasileiros de Finanças parecem alinhar-se com a realidade nacional quanto aos estímulos do sistema de avaliação da Capes. São prováveis reflexos positivos dessa avaliação: (1) o crescimento no número de autores, prolíficos e em geral; (2) o estímulo a parcerias entre autores, que pode aumentar a qualidade dos artigos; (3) o aumento no número de artigos e de periódicos nacionais de boa qualidade, com mais especialização; (4) e a maior quantidade de artigos publicados no exterior e em inglês. A evidência do presente artigo também sugere consequências negativas desses estímulos, tais como o aumento de artigos com mais de três autores e a grande quantidade de publicações em periódicos internacionais de qualidade muito baixa. O possível aspecto negativo do crescimento da coautoria é a tentativa de somar mais pontos para os programas envolvidos ao se colocarem, por exemplo, todos os membros de uma banca como coautores. O número de artigos com dois e três autores superou o de artigos com apenas um autor e é um resultado novo em relação a um estudo anterior.

\section{Propostas para o desenvolvimento da área}

Uma aspiração para o maior desenvolvimento da área é o aumento da quantidade de artigos publicados em periódicos internacionais de Finanças de alto impacto. É preciso, porém, que os artigos desenvolvam modelos teóricos e que apresentem análises empíricas que avancem o conhecimento global e que não se limitem a replicar exercícios empíricos ao Brasil. Contudo, os estímulos oferecidos pelo sistema de avaliação da Capes na área de Administração podem estar aquém do necessário para que esse passo seja dado. Um artigo do estrato A1 vale 100 pontos, enquanto artigos do estrato A2 e B1, em que se encontram os periódicos aqui analisados, valem, respectivamente, 80 e 60 pontos. Além disso, muitos dos periódicos internacionais de Finanças classificados como A1 são considerados, de fato, ótimos periódicos de segunda linha por universidades estrangeiras de ponta. A experiência dos autores sugere que é mais fácil publicar dois ou três artigos A2 ou B1 do que um artigo em um dos periódicos mais notáveis.

Incentivar as redes internacionais de pesquisadores, os doutoramentos em universidades de ponta nos EUA, especialmente, e a visita de pesquisadores internacionais de primeira linha é crucial para que jovens pesquisadores em Finanças adquiram as habilidades necessárias para construírem modelos teóricos, tenham acesso às caríssimas fontes de dados internacionais e participem de parcerias que incluam autores que publiquem nos periódicos internacionais notáveis de Finanças. Finalmente, a criação de um estrato "A1+", com pontuação elevada e contendo somente os periódicos considerados notórios por pesquisadores internacionais de renome, e não por um comitê formado por autores vinculados a instituições nacionais, seria um estímulo importante, na nossa opinião. É de nosso conhecimento que algumas instituições já concedem prêmios financeiros elevados para os que publicam nesses periódicos "A1+".

Atualmente, parece haver dois "mundos": o dos pesquisadores de Finanças vinculados às principais instituições internacionais, que competem para ver seus artigos publicados nos melhores periódicos científicos mundiais da área, e o dos pesquisadores nacionais, que concorrem para publicar seus artigos nos melhores periódicos científicos nacionais, que oferecem quase o mesmo número de pontos que os primeiros, mas são desconhecidos no cenário mundial. O sistema de avaliação poderia aproximar os dois "mundos", pois, observando os resultados aqui obtidos, a produção da área no País ainda não reflete tanto a amplitude quanto a profundidade da inovação teórica e metodológica observáveis nos países mais desenvolvidos.

\section{REFERÊNCIAS}

BERTERO, C. O; CAlDAS, M. P; WOOD JR, T. (Org). Produção científica em administração no Brasil: o estado da arte. São Paulo: Atlas, 2005. 
BOROKHOVICH, K. A; BRICKER, R. J; SIMKINS, B. J. Journal communication and influence in financial research. The Journal of Finance, v. 49, n. 2, p. 713-725, 1994.

BREALEY, R. A; MYERS, S. C; ALLEN, F. Principles of corporate finance. Boston: McGraw-Hill Irwin, 2006.

CAMARGOS, M. A; COUTINHO, E. S; AMARAL, H. F. O perfil da área de Finanças do Enanpad: um levantamento da produção cientifica e de suas tendências entre 2000-2004. In: ENCONTRO NACIONAL DA ASSOCIAÇÃO NACIONAL DE PROGRAMAS DE PÓS-GRADUAÇÃO E PESQUISA EM ADMINISTRAÇÃO, 29, 2005, Brasília. Anais. Rio de Janeiro: ANPAD, 2005.

CHUNG, K. H; COX, R. A. K. Patterns of productivity in the finance literature: a study of the bibliometric distributions. The Journal of Finance, v. 45, n. 1, p. 301-309, 1990.

CURRIE, R. R; PANDHER, G. S. Finance journal rankings and tiers: an active scholar assessment methodology. Journal of Banking E Finance, v. 35, n. 1, p. 7-20, 2011.

IQUIAPAZA, R. A; AMARAL, H. F; BRESSAN, A. A. Evolução da pesquisa em Finanças: epistemologia, paradigma e críticas. Organizações E Sociedade, v. 16, n. 46, p. 351$370,2009$.

LEAL, R. P. C; OLIVEIRA, J. de; SOLURI, A. F. Perfil da pesquisa em Finanças no Brasil. RAE-Revista de Administração de Empresas, v. 43, n. 1, p. 91-104, 2003.

MATSUMOTO, A. S; TABAK, B. M; CAJUEIRO, D. O; BRITO, J. C; SOUZA, P. A. A pesquisa em Finanças no Brasil: a estrutura das colaborações científicas. In: SIMPÓSIO DE EXCELÊNCIA EM GESTÃO E TECNOLOGIA - SEGeT, 5, 2008, Resende. Anais. Resende: Associação Educacional Dom Bosco, 2008.

SOUZA, F. C. de; MURCIA, F. D; BORBA, J. A. Doutorados em Finanças no Brasil e nos Estados Unidos: percepções do corpo docente relativas ao ensino e pesquisa na área. Revista de Administração da Unimep, v. 8, n. 3, p. 161183, 2010. 